\title{
Reply to Letter to the Editor by Pandher and Boparai (MS 08-00749)
}

\author{
Kung-Chia Li MD, PhD
}

Published online: 15 August 2008

(C) The Association of Bone and Joint Surgeons 2008

\section{Dear Sirs:}

I appreciate your interest and comments regarding the article by $\mathrm{Li}$ et al. [1]. I received numerous comments about this paper. I and the other authors of that article believe more discussion and critical review will aid progress.

In response to your concerns, first, delaying mobilization is against our principles. Our postoperative protocol is based on mobilizing the patient as early as possible. Approximately $80 \%$ of our patients with Frankel E and D spinal injuries walked within 6 to 8 hours after surgery, and the remaining $20 \%$ after 8 to 24 hours. We encourage patients to walk as soon and as much as possible. We also believe that most burst fractures without neural deficits will not need any surgery if patients can stay in bed for 3 months to allow bone and disc healing.

We believe short-segment fixation with TpBA augmentation is the gold standard for one burst fracture. We believe long-segment fixation is unnecessary for most burst fractures and we do not use it. Therefore, we would be unable to follow your suggestion to compare results of long-segment fixation with TpBA augmentation. Ideally, a single vertebral body problem should be treated over the lesion site alone; we believe fusion involving an intact vertebra is not logical and unnecessary and should be avoided if possible. I predict the trend to treat burst fractures will be long-segment fixation, then short-segment fixation with anterior support, and finally only vertebral reconstruction.

With practice, blood loss and operating time can be limited. After acquiring experience with the TpBA technique, inserting one TpBA is no more than one pedicle screw.

We used dynamic views to check the spine, including stability of the TpBA and status of adjacent discs or vertebrae.

Finally, the failure in one of our patients occurred during the early learning curve and the pedicle cortex problem was neglected at primary surgery. At revision surgery we noted the inferior cortex of the pedicle had been violated. More than 5000 TpBA vertebroplasties have now been performed at our hospital and such problems with pedicle rupture essentially have been eliminated.

\section{Reference}

1. Li KC, Hsieh $\mathrm{CH}$, Lee $\mathrm{CY}$, Chen TH. Transpedicle body augmenter: a further step in treating burst fractures. Clin Orthop Relat Res. 2005;436:119-125.
Re: Transpedicle body augmenter: a further step in treating burst fractures.

K.-C. Li ( $\square)$

Institute and Faculty of Physical Therapy, National Yang-Ming University, Chia-Yi Yang-Ming Hospital, Chia-Yi 112, Taiwan, ROC

e-mail: kungchiali@gmail.com 